\title{
Fî́g:
}

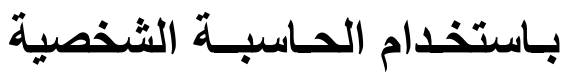

د. قيدار مجيـد قبـع

جامعة الموصل-كلية هندسة الإكترونيات
رغد غالب سعد الله السلطان

جامعة الموصل-كلية الهندسة الهبطة

الخلاصة

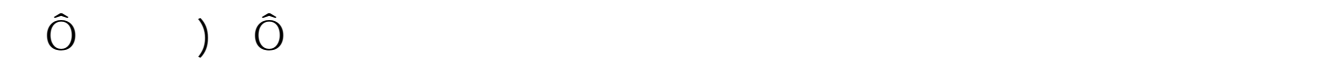

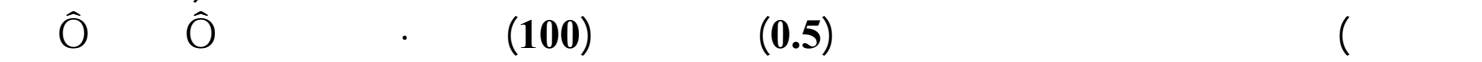

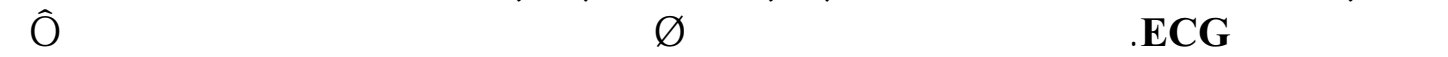

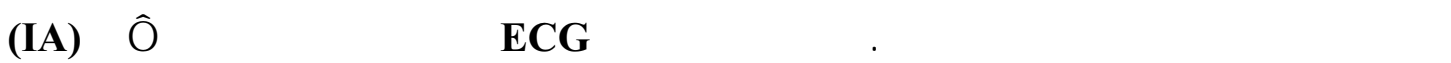

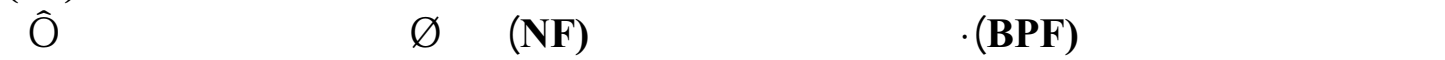

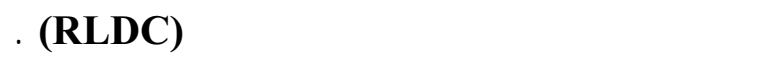

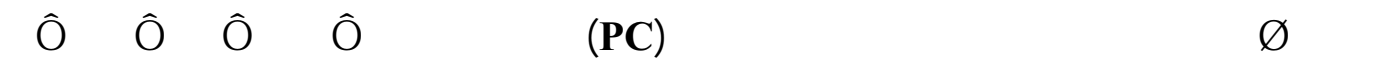

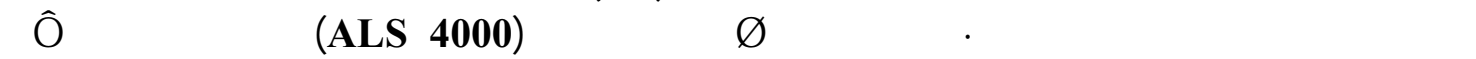

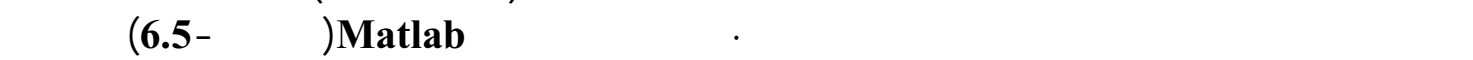

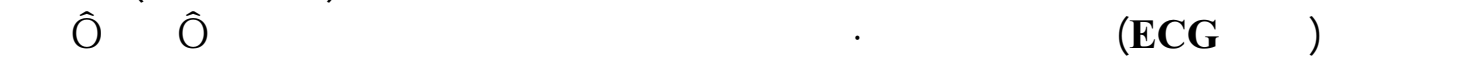

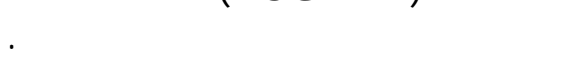

\section{DESIGN AND IMPLEMENTATION OF A SINGLE- CHANNEL ECG MONITOR USING PC}

\author{
Raghad G. AL-Sultan \\ University of Mosul \\ College of Engineering
}

\author{
Dr.Kaydar M. Quboa \\ University of Mosul \\ College of Electronics
}

\section{ABSTRACT}

An Electro-Cardio-Graph (ECG) is a device that records the electrical activity of the heart. Skin electrodes placed at designated locations on the body collect ECG measurement information, and from these waveforms different information on heart condition can be derived.

This paper discusses the design and implementation of a single-channel ECG (three-electrode) circuit with a bandwidth of (0.5-100) Hz for patient monitoring purposes. The front end of an ECG must be able to deal with weak signals ranging from few microvolts to few millivolts. An analogue ECG amplifier is designed and implemented using instrumentation amplifier (IA) followed by band pass filter (BPF), then by notch filter (NF) to minimize noise affecting the signal, and a right leg drive circuit (RLDC). .

The ECG data is recorded and analyzed with greater flexibility using personal computer. The (ALS 4000) PC-sound card is used as a data acquisition device. The Matlab program (version 6.5) is employed to acquire data from sound card then digitally processing the signal in order to display the ECG signal on a PC monitor. 


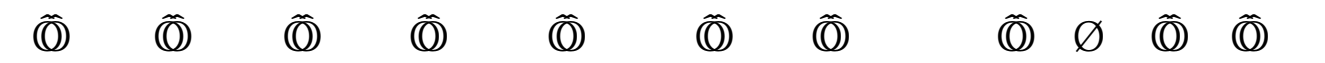

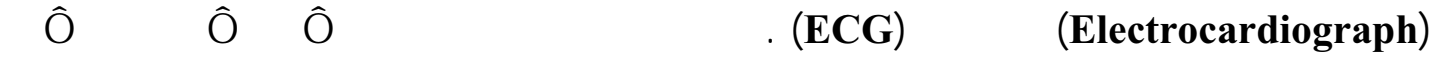

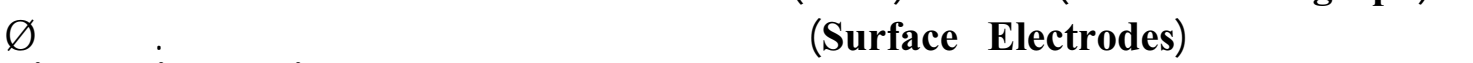

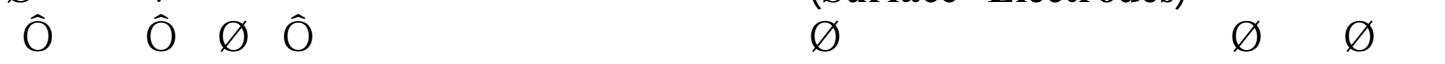

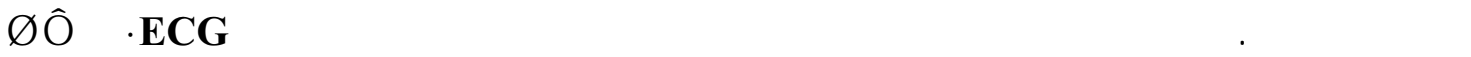
rof fữ

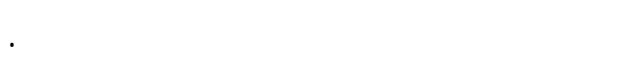

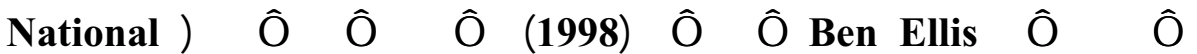

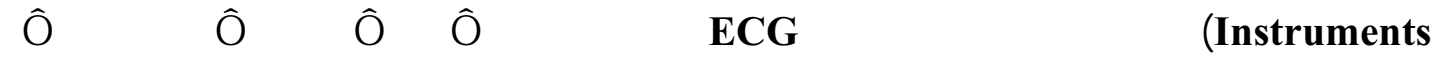

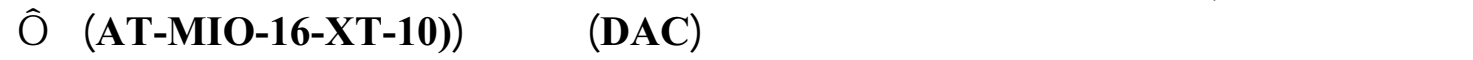

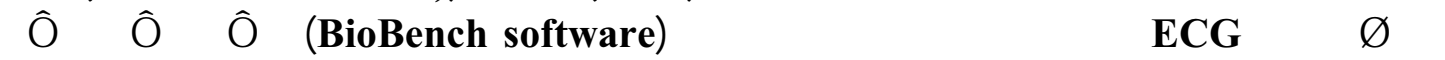

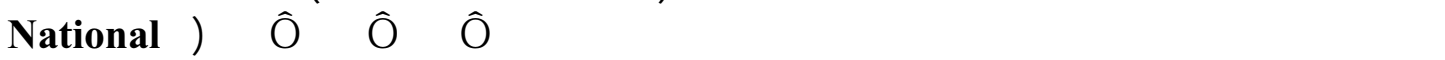
.[1]

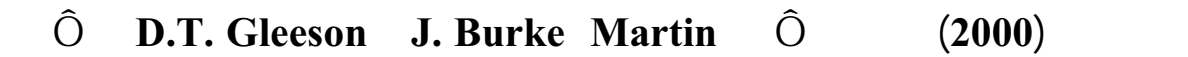

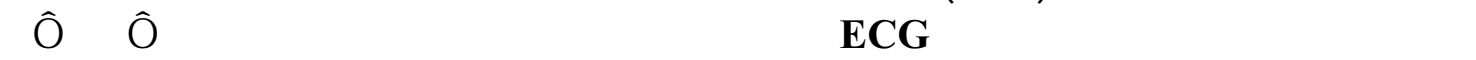

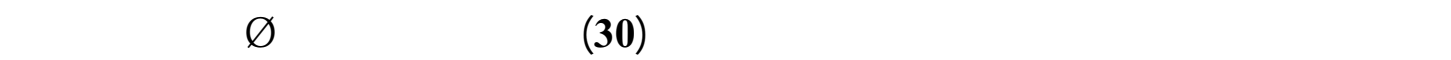

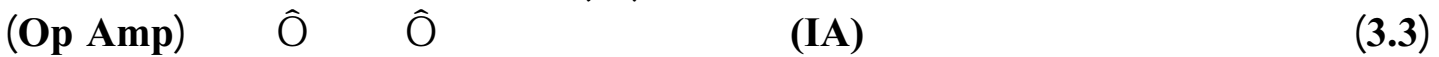
. [2](MAX400) ugé

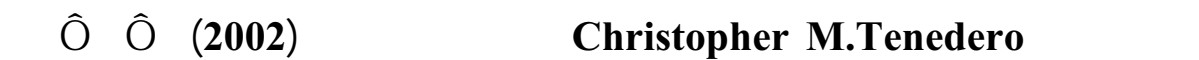

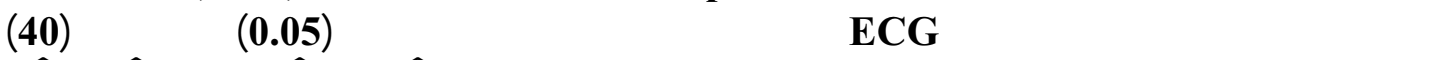

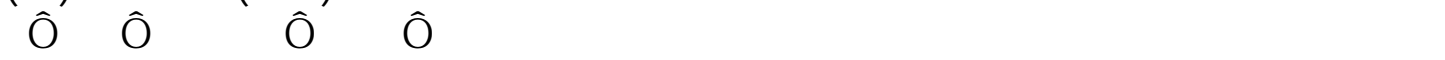

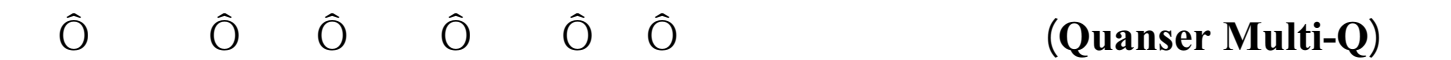

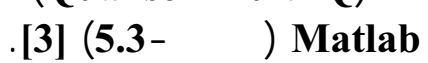

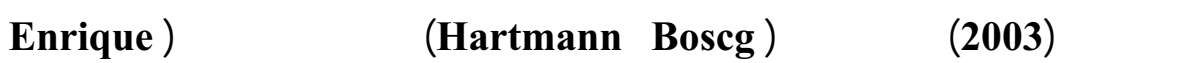

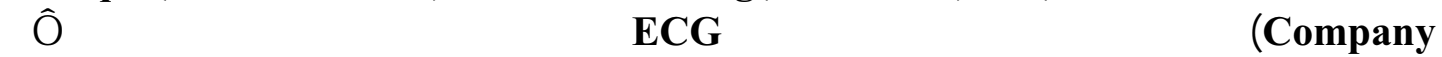

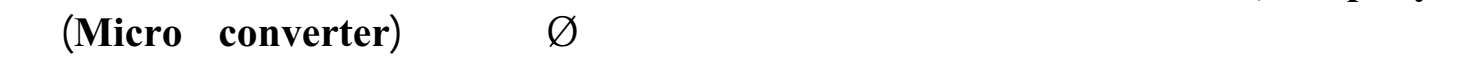

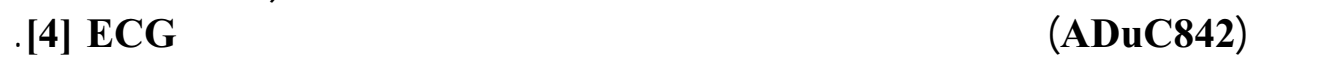

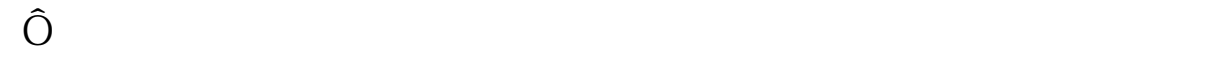

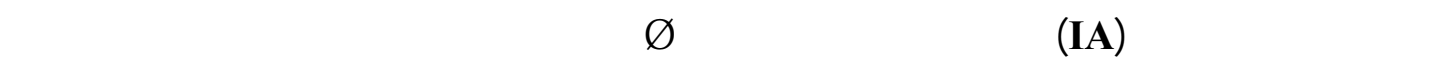

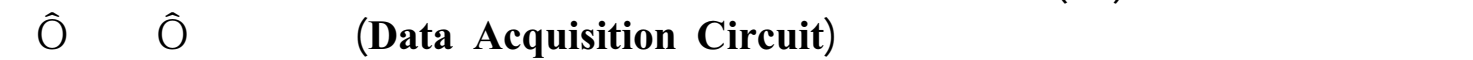

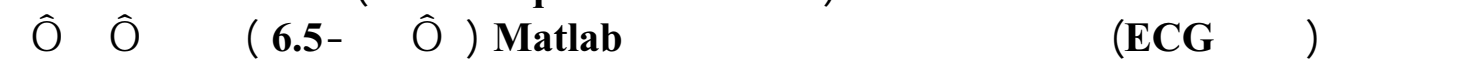

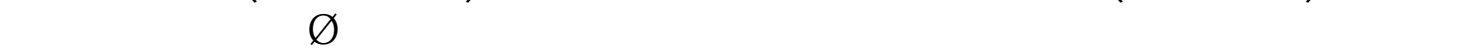

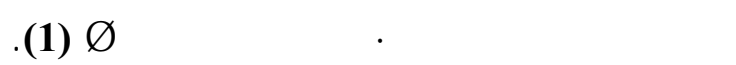




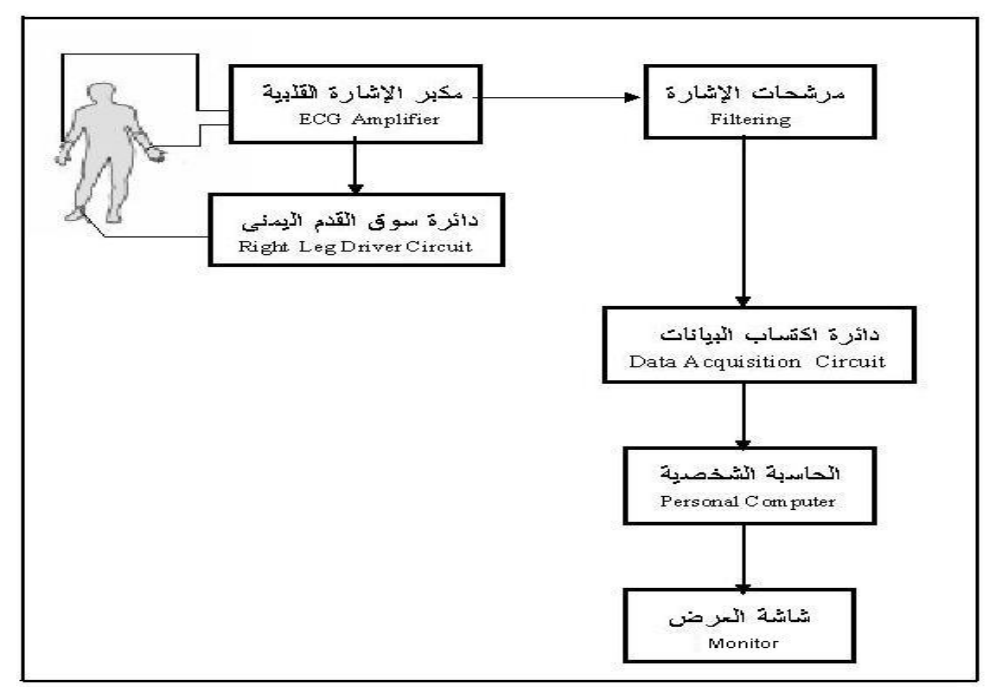

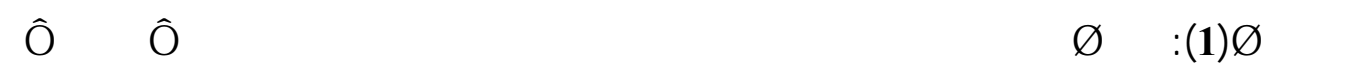

Ŕgǚ́n:

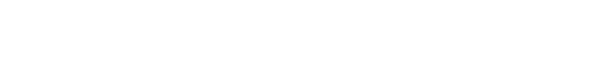

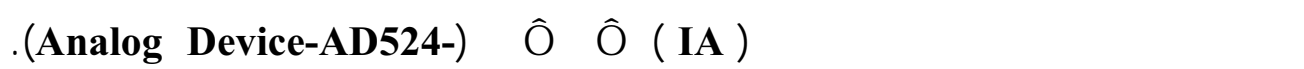

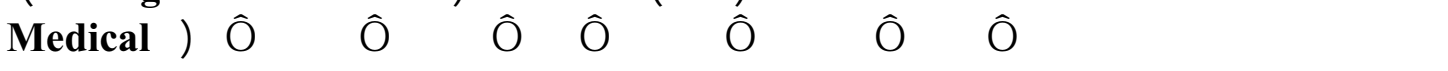

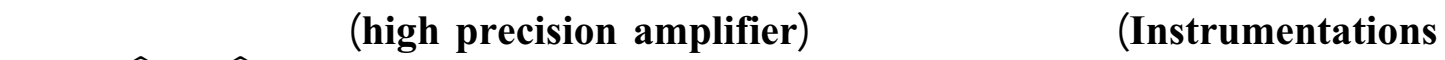

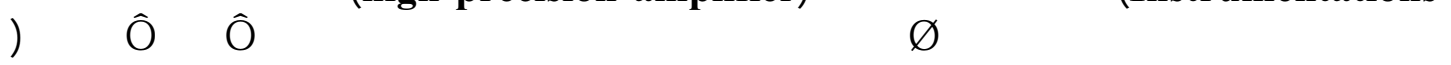

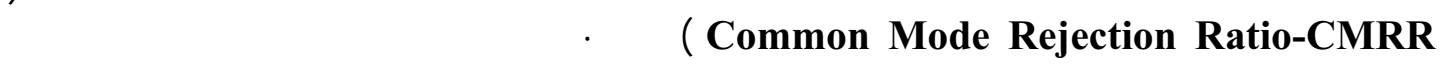

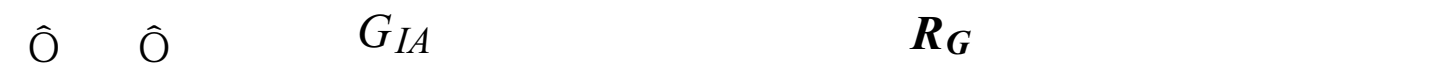

$$
G_{I A}=\frac{40 K}{R_{G}}+1
$$

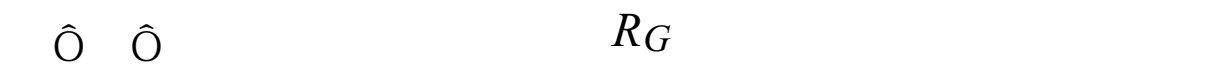

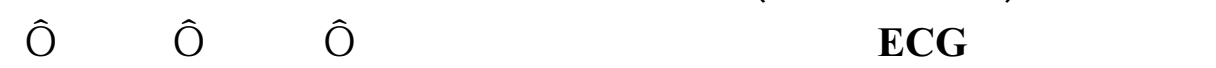

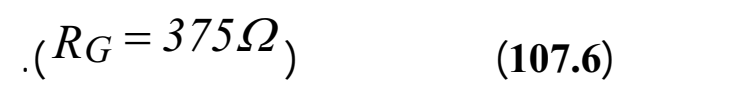

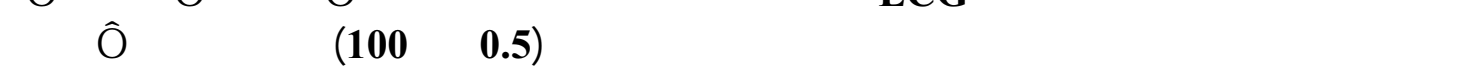

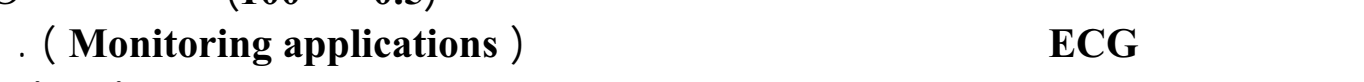

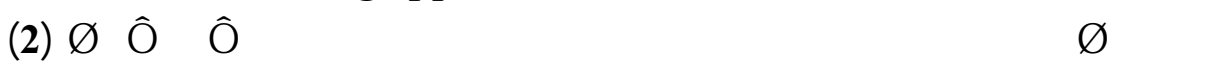

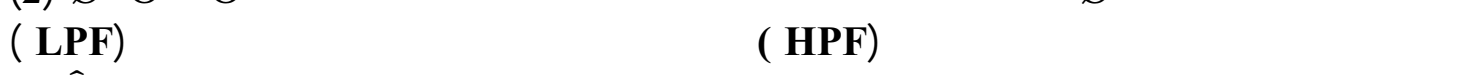

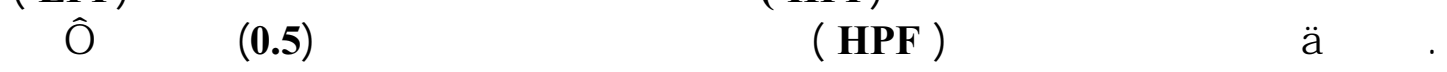

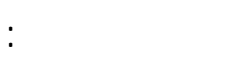

$$
f_{h c}=\frac{1}{2 \pi R_{i} C_{i}}
$$




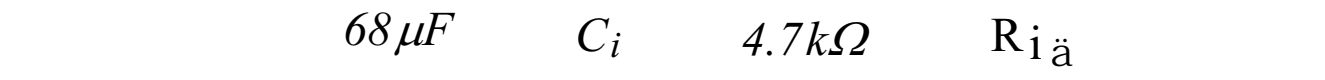

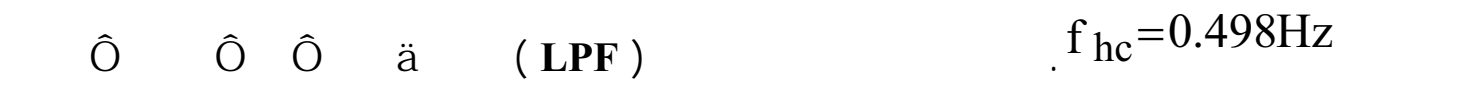

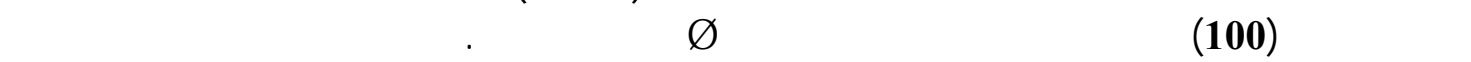

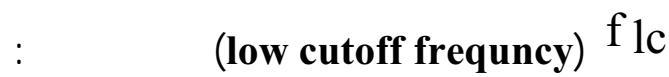

$\mathrm{flc}_{\mathrm{lc}}=\frac{1}{2 \pi \mathrm{R}_{\mathrm{f}} \mathrm{C}_{\mathrm{f}}}$

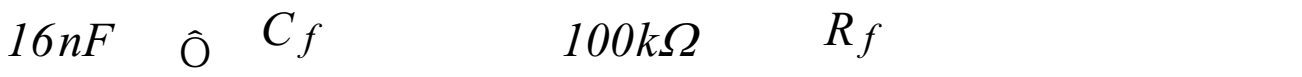

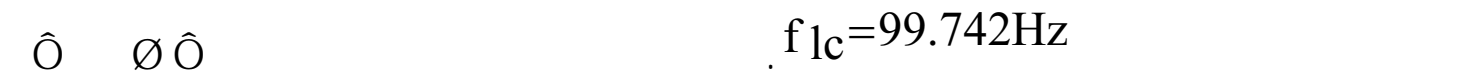

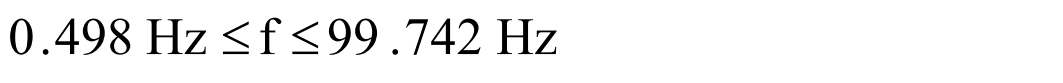

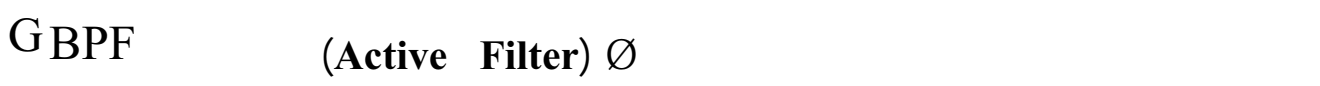
: Egdef $\mathrm{GBPF}=\frac{\mathrm{R}_{\mathrm{f}}}{\mathrm{R}_{\mathrm{i}}}=\frac{100^{*} 10^{3}}{4.7 * 10^{3}}=21.276$

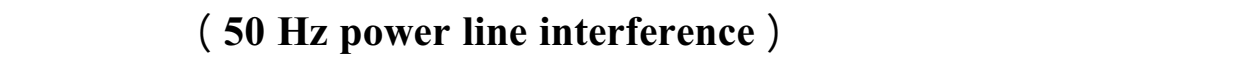

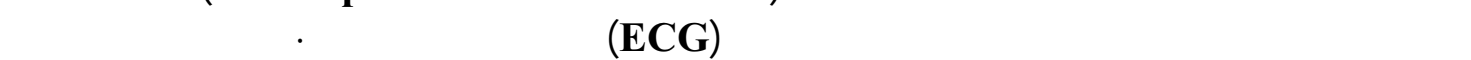

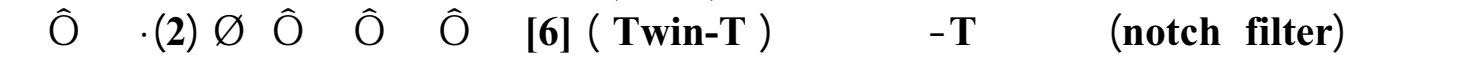

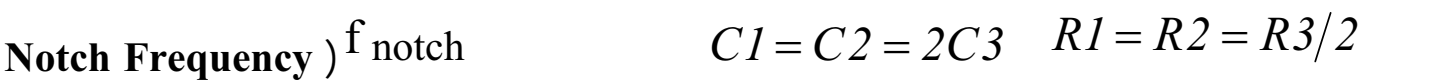

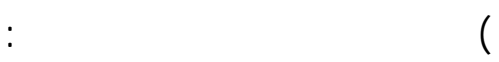
$\mathrm{f}$ notch $=\frac{1}{2 \pi \mathrm{R}_{2} \mathrm{C}_{2}}$

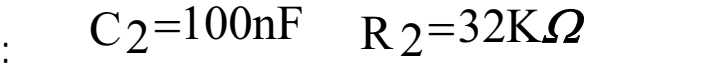

$$
\begin{aligned}
& \mathrm{f}_{\text {notch }}=\frac{1}{2 * \pi * 32 * 10^{3 * 100 * 10^{-9}}}=49.76 \mathrm{~Hz}
\end{aligned}
$$

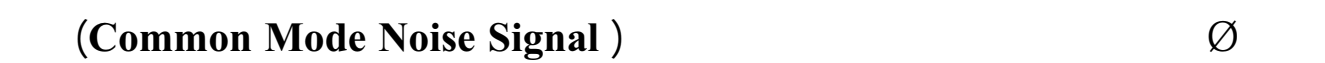

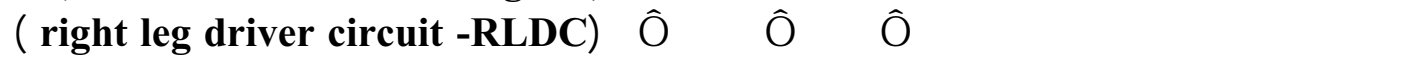

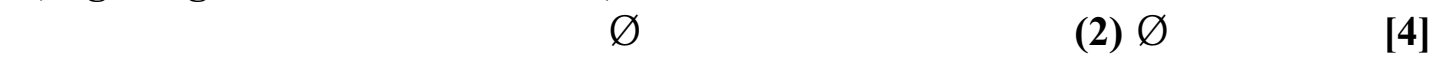

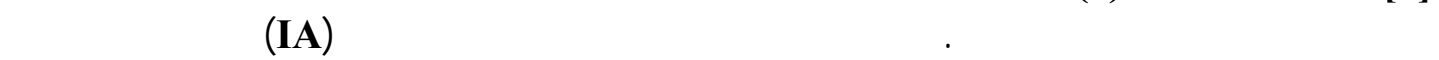

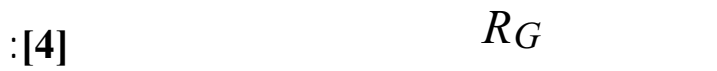

$$
R_{G}=R b / /(2 * R a)
$$




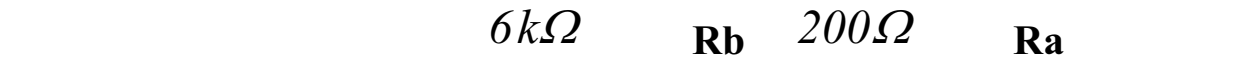

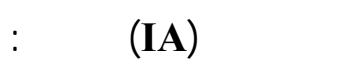

$$
R_{G}=6 \mathrm{k} \Omega / /(2 * 200)=375 \Omega
$$

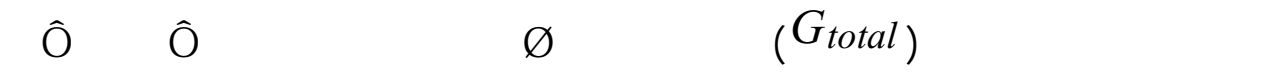

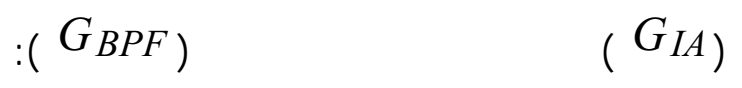

$$
G_{\text {total }}=G_{I A}{ }^{*} G_{B P F}
$$

$$
\therefore G_{\text {total }}=107.3 * 21.276=2282.9148
$$

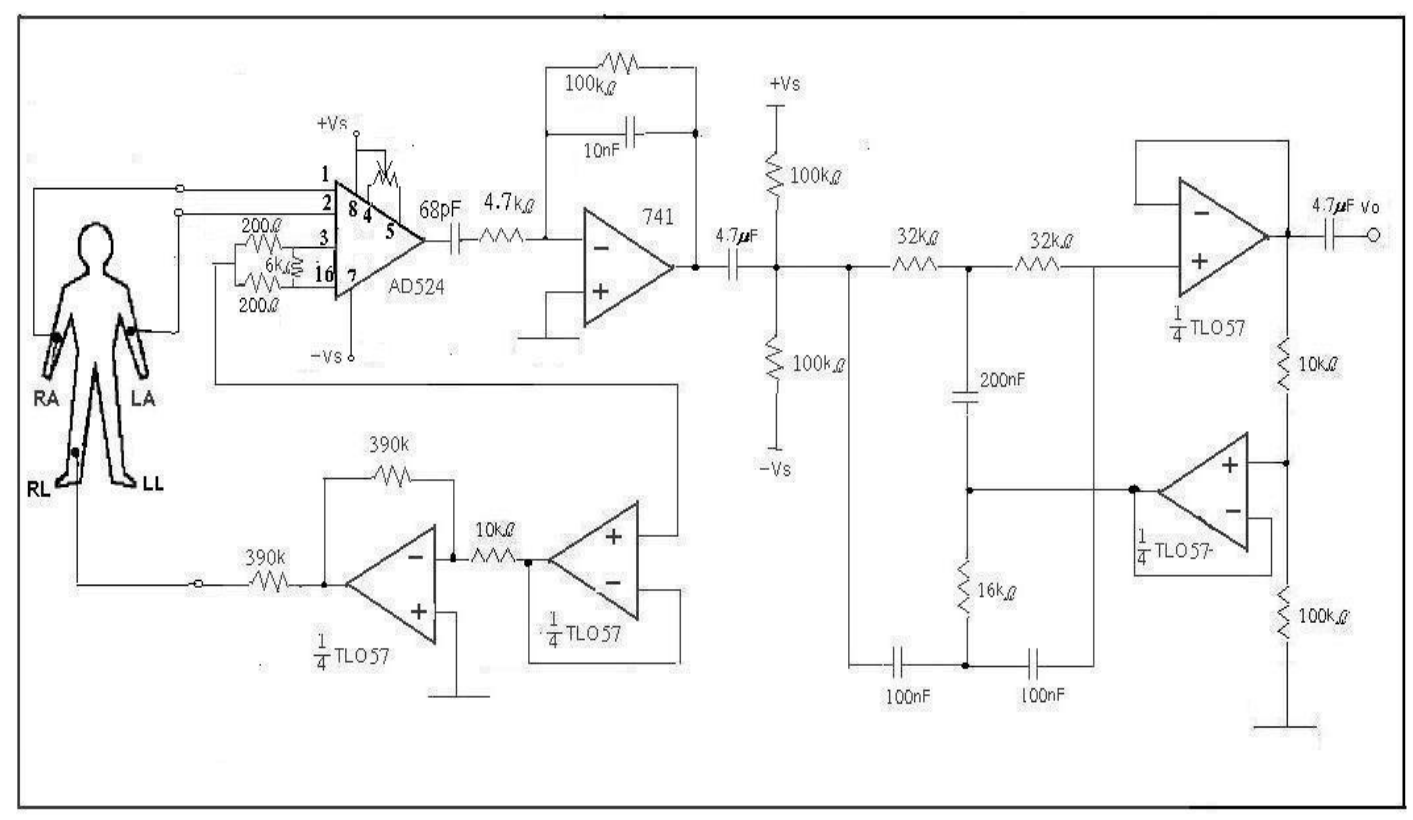

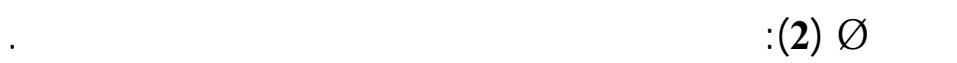

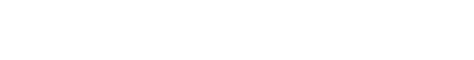

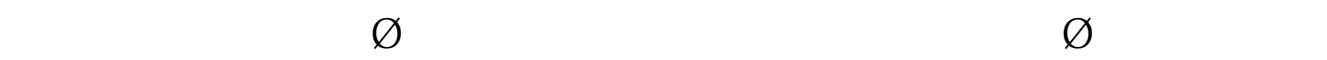

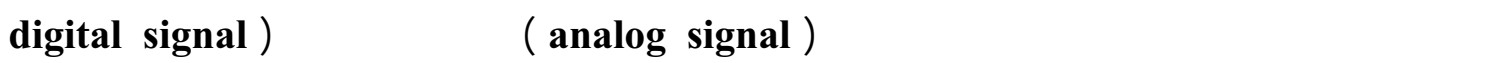

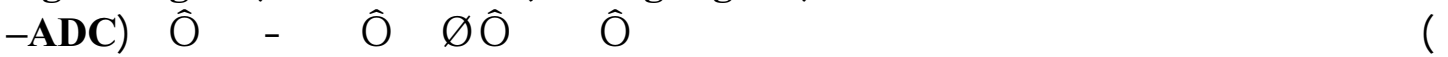

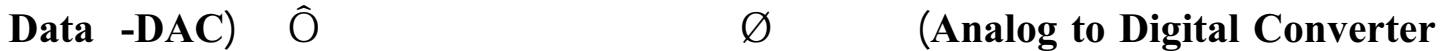

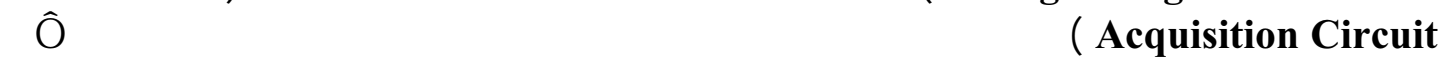

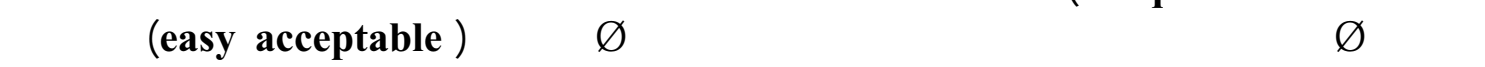

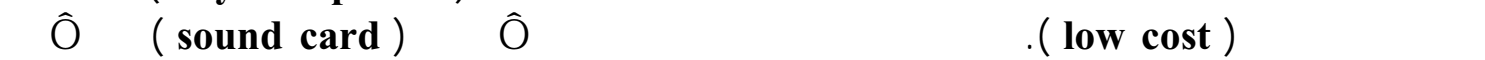




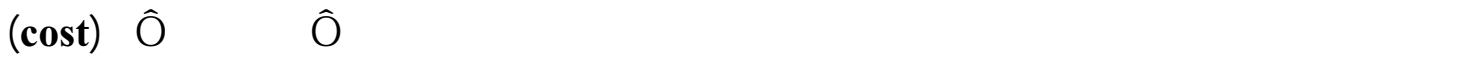

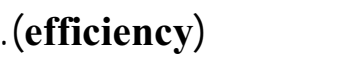

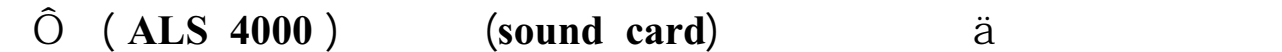

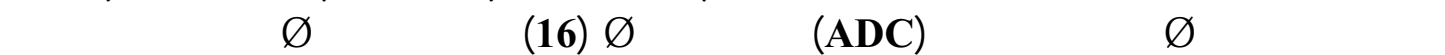

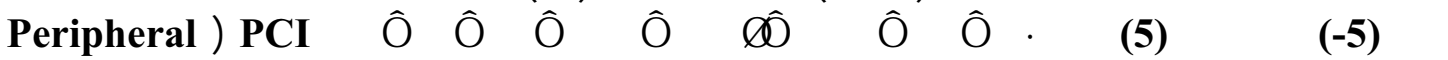

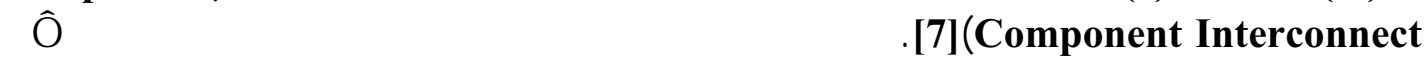

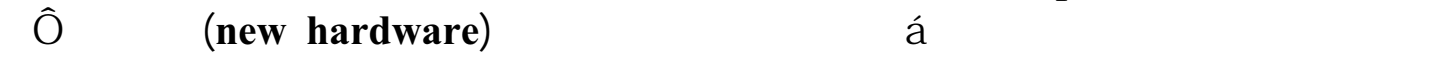

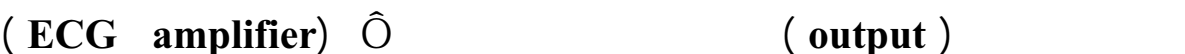

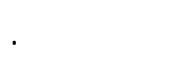

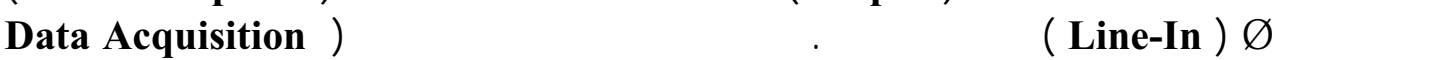

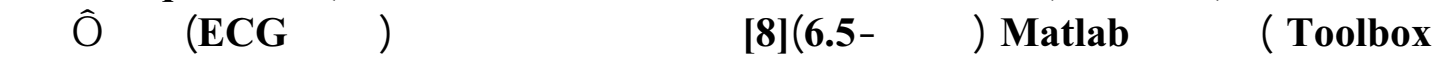

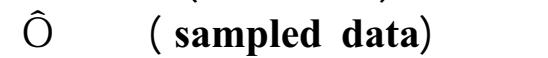

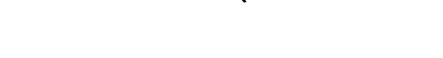

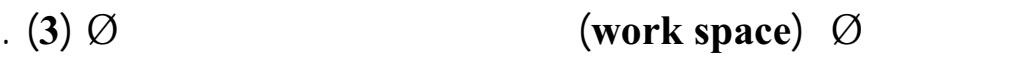

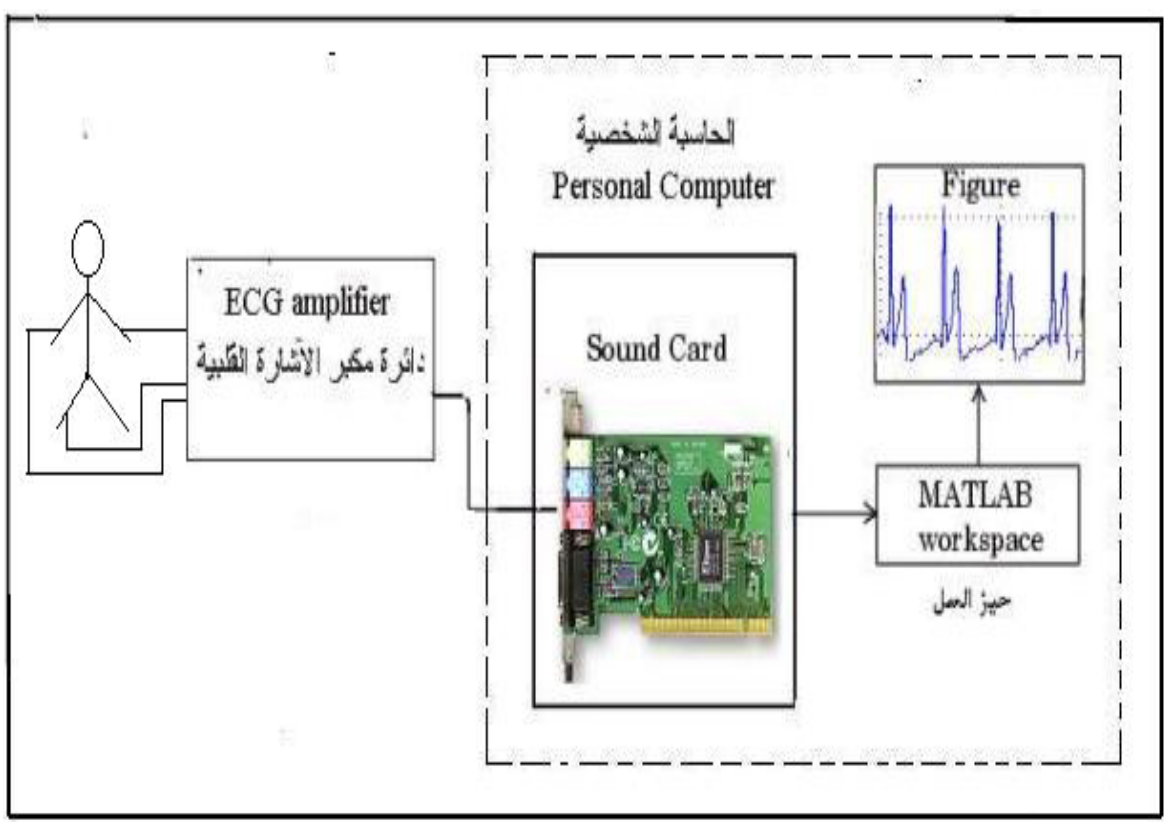

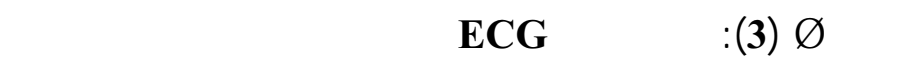

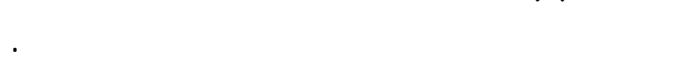

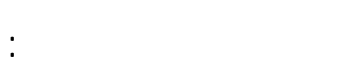

[S fs]=daqrecord ('time', 'sampling frequency')

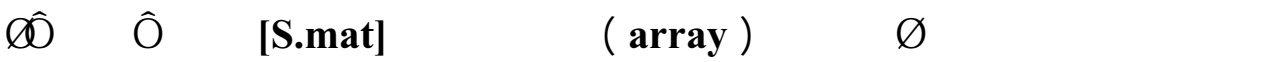

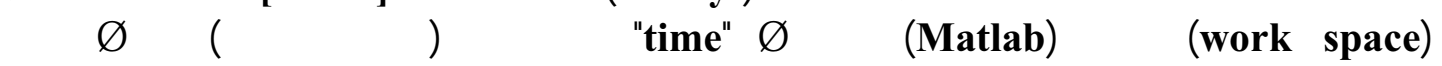

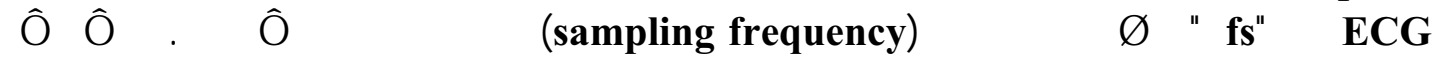

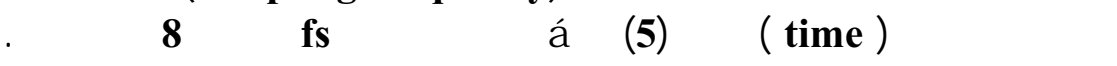




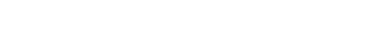

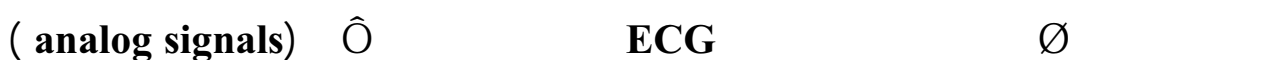

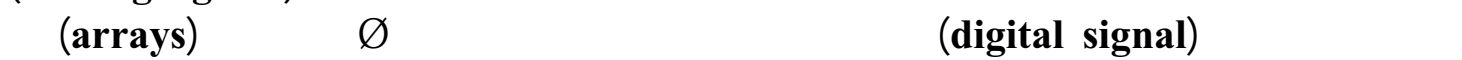

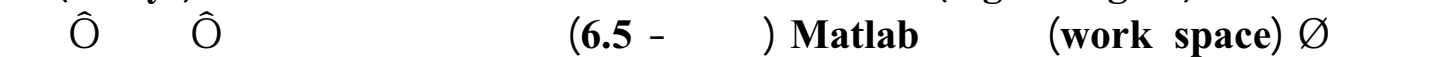

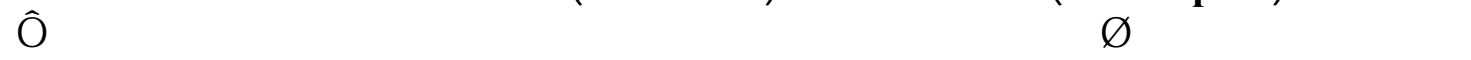

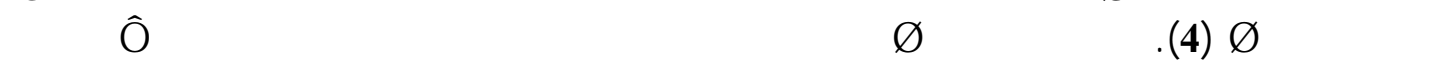

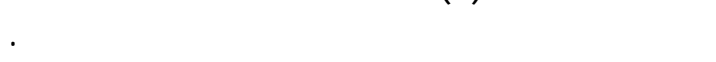

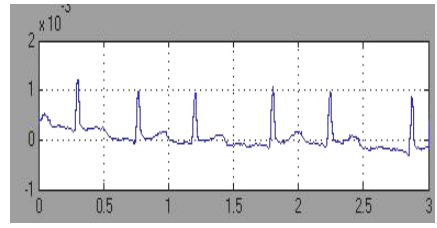

$-3-$

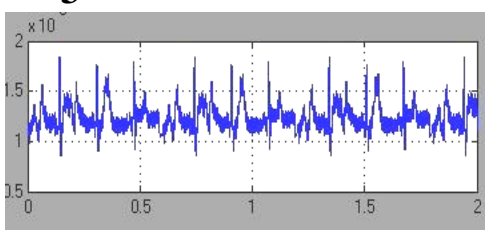

$-6-$

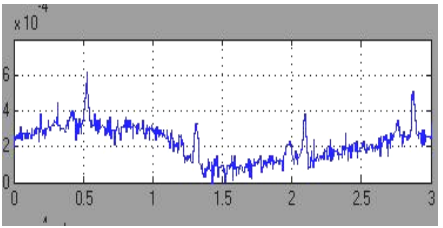

$-2-$

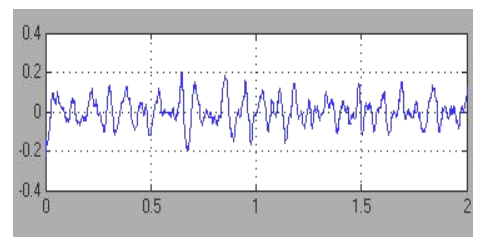

$-5-$

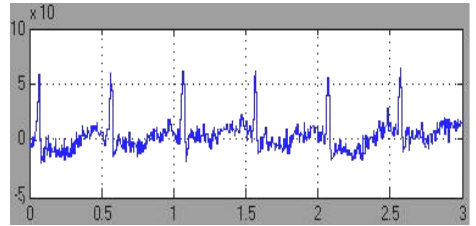

$-1-$

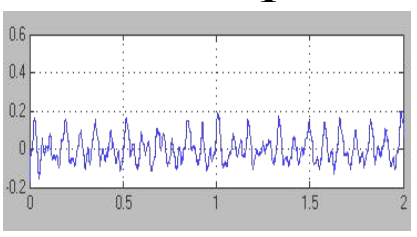

$-4-$

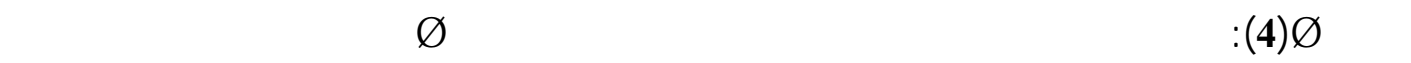

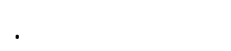

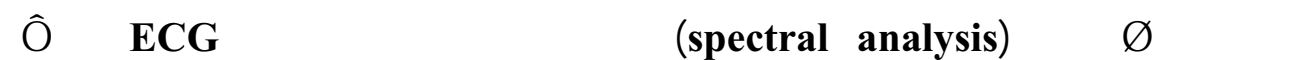

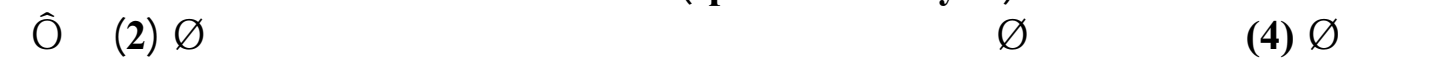

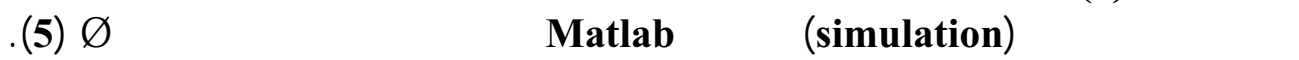
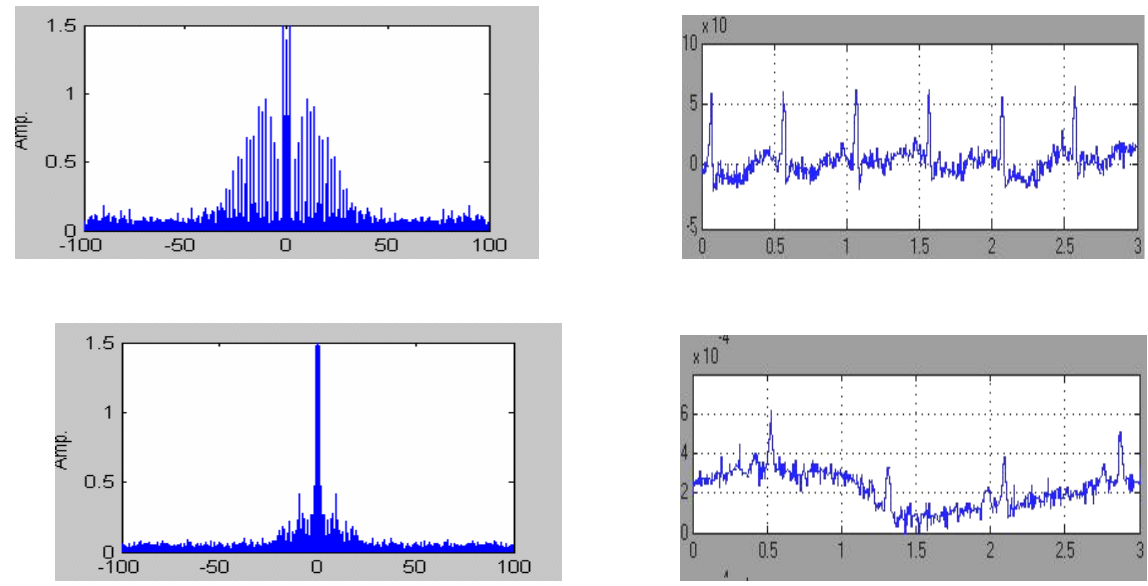

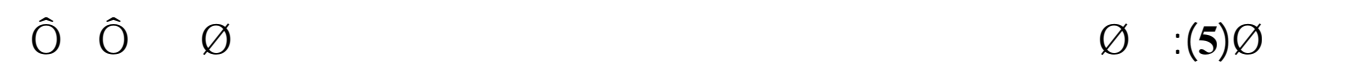

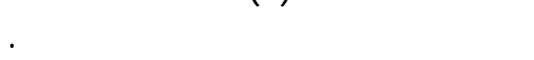




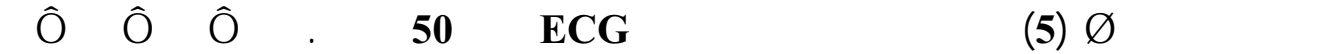

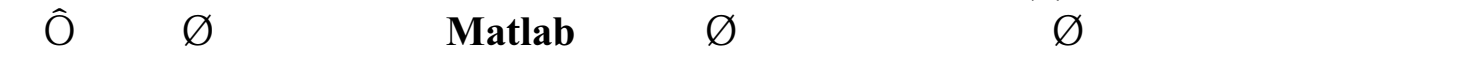

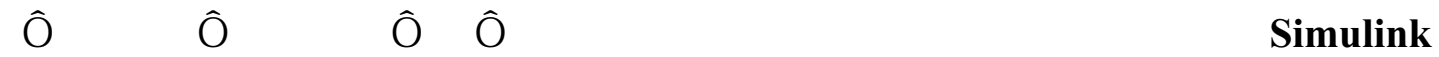

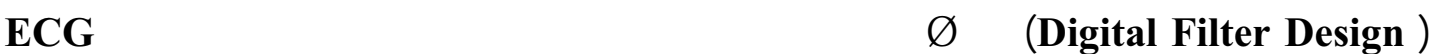

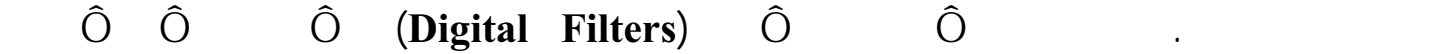

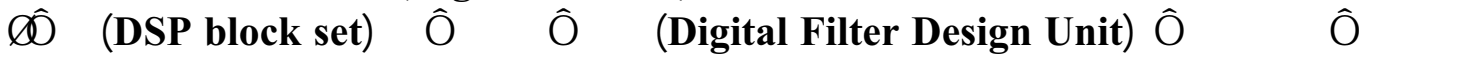

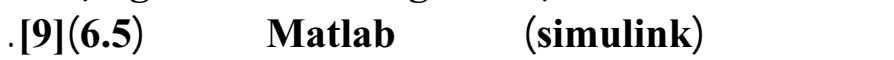

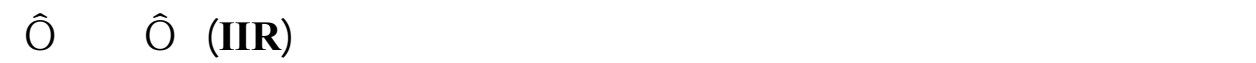

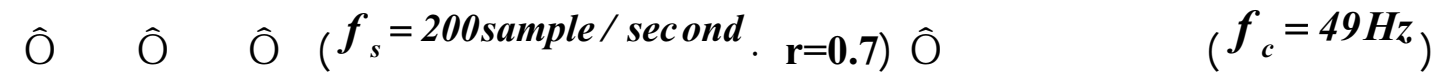

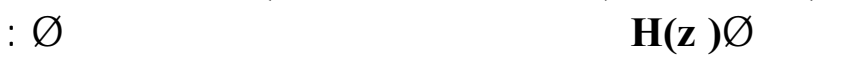

$$
H(Z)=\frac{1+2 Z^{-1}+Z^{-2}}{1-0.043975 Z^{-1}+0.49 Z^{-2}}
$$

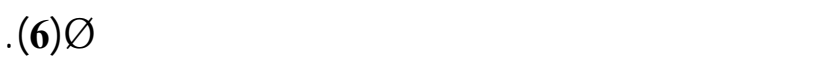

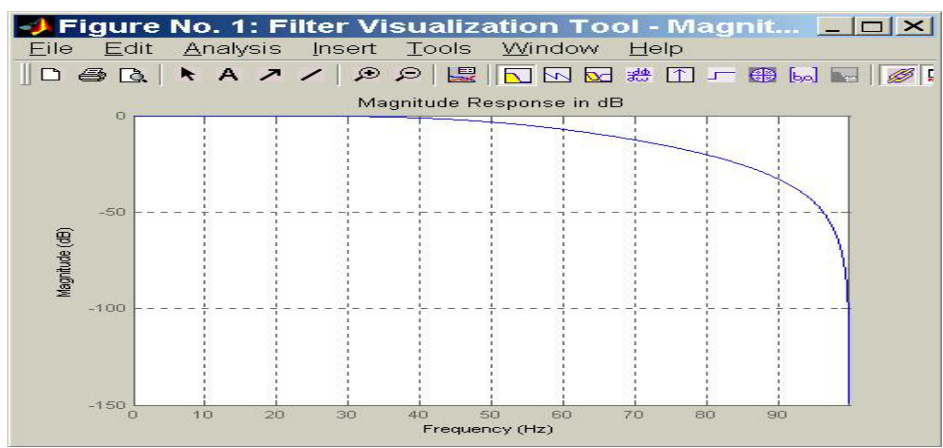

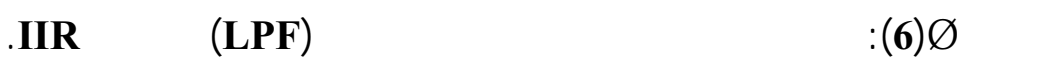

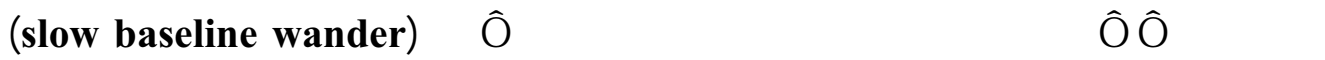

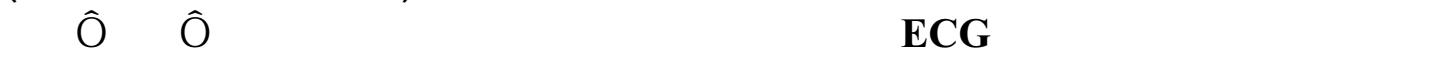

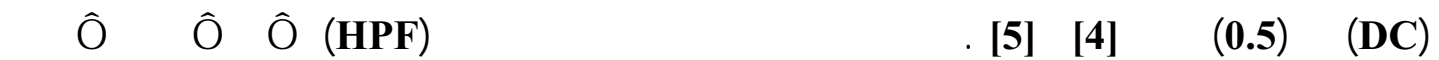

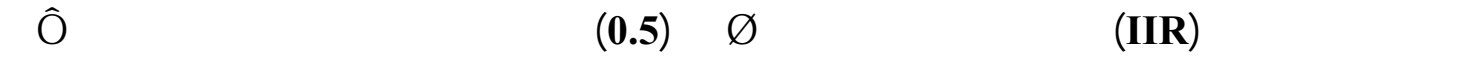

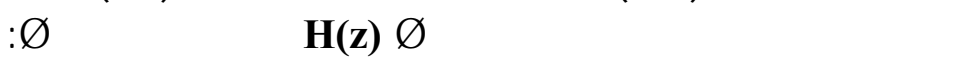

$$
H(Z)=\frac{1-2 Z^{-1}+Z^{-2}}{1-1.3998272 Z^{-1}+0.49 Z^{-2}}
$$

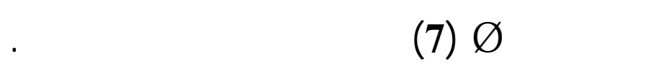




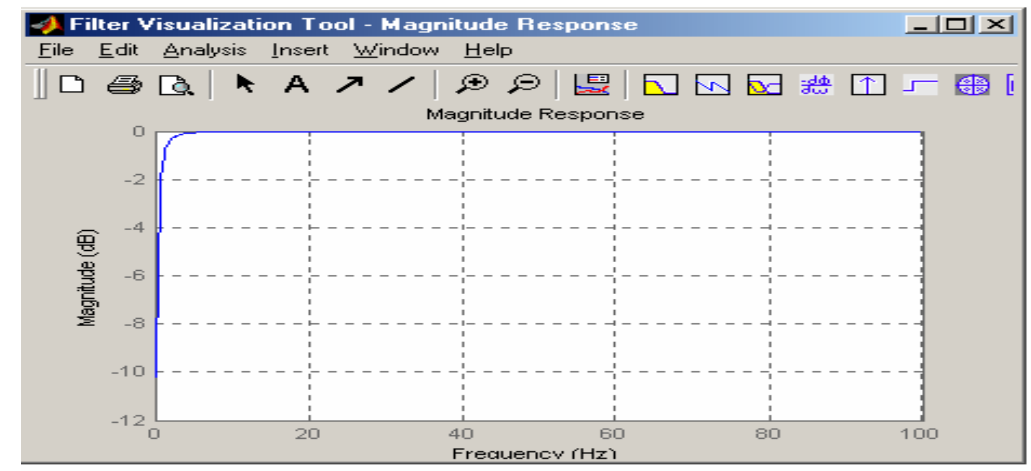

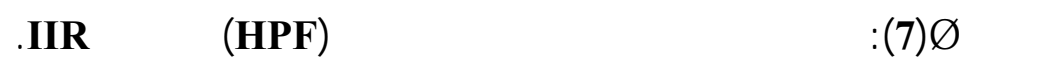

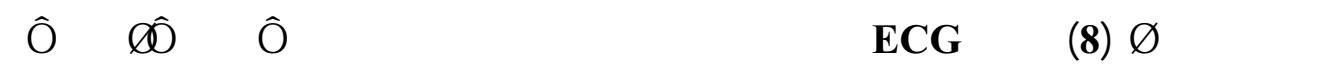

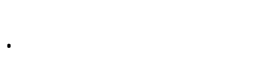

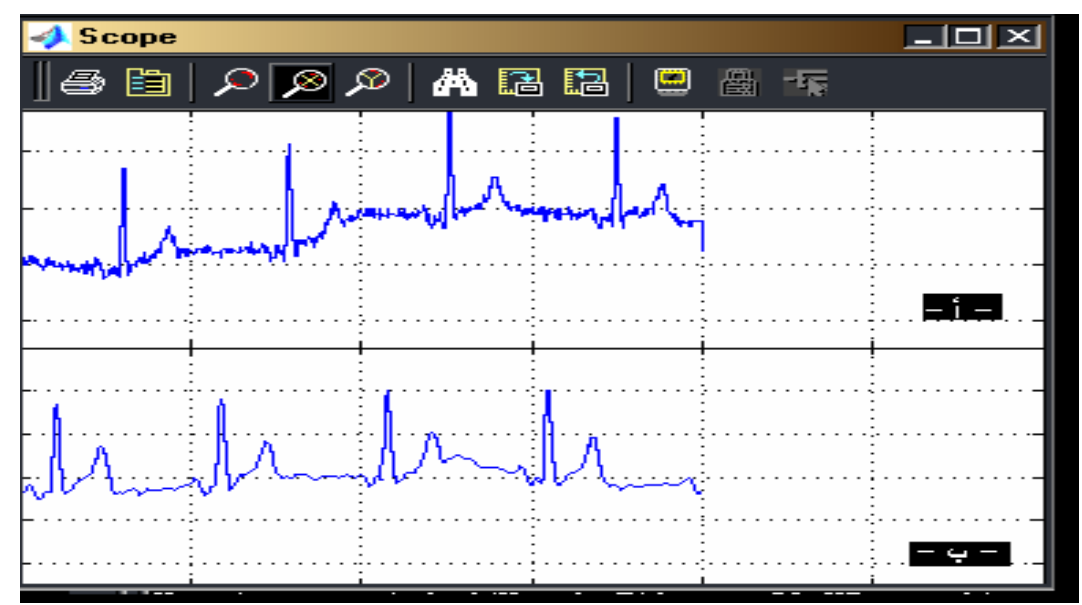

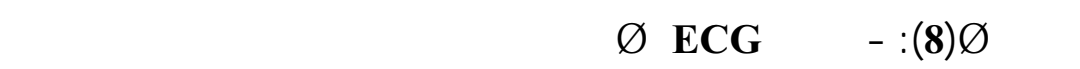

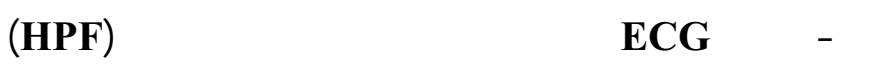

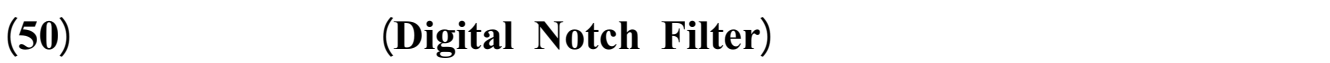

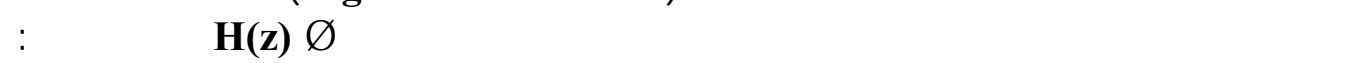
$\mathrm{H}(\mathrm{z})=\frac{1+\mathrm{Z}^{-2}}{1+0.49 \mathrm{Z}^{-2}}$

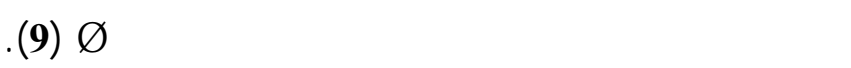




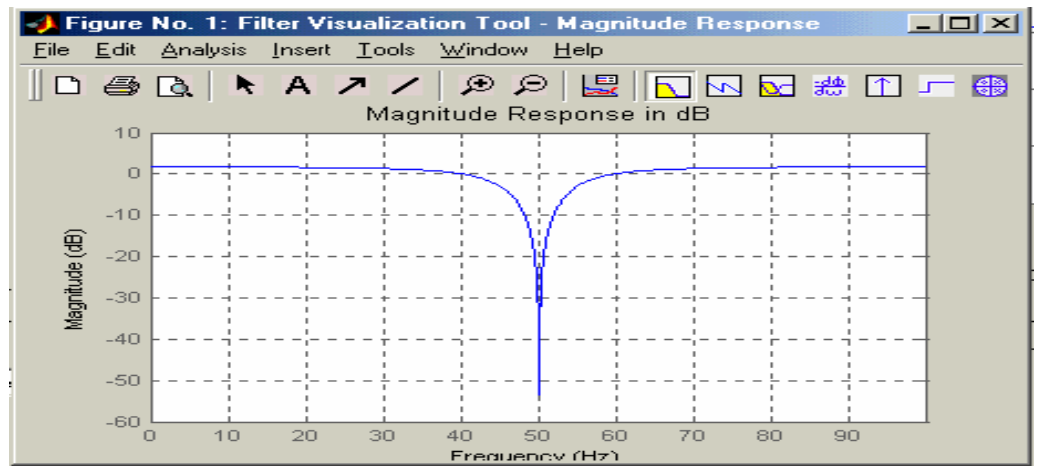

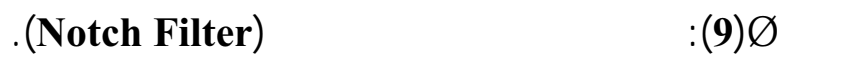

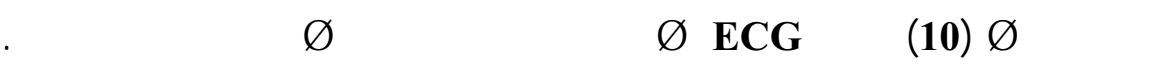

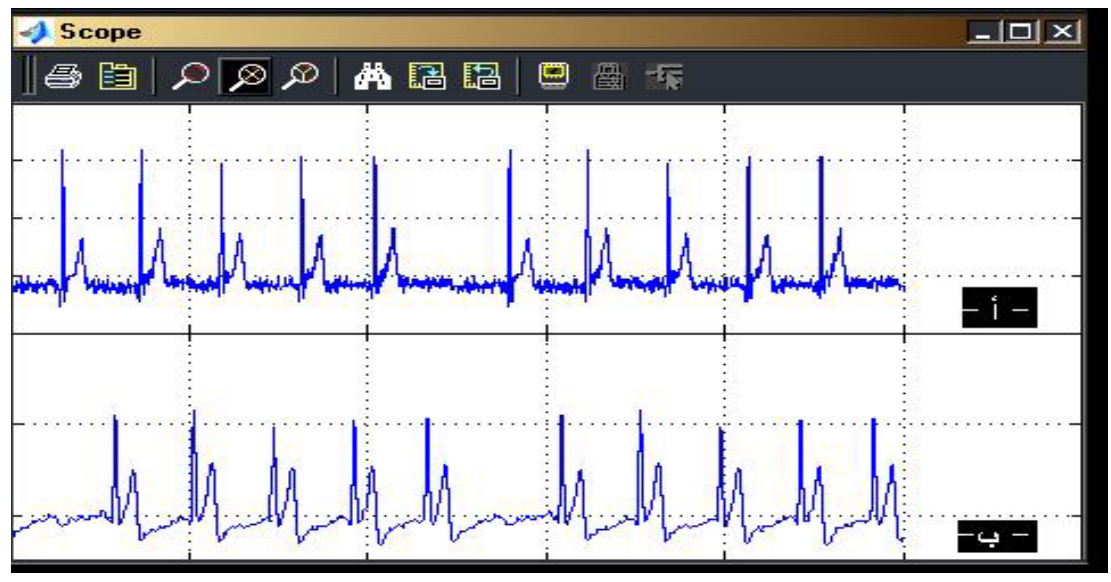

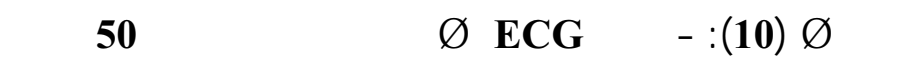

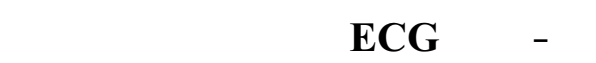

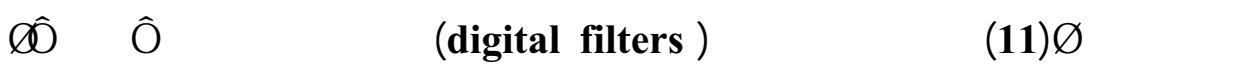

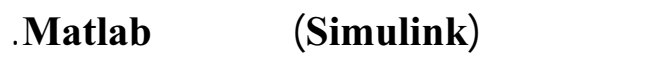




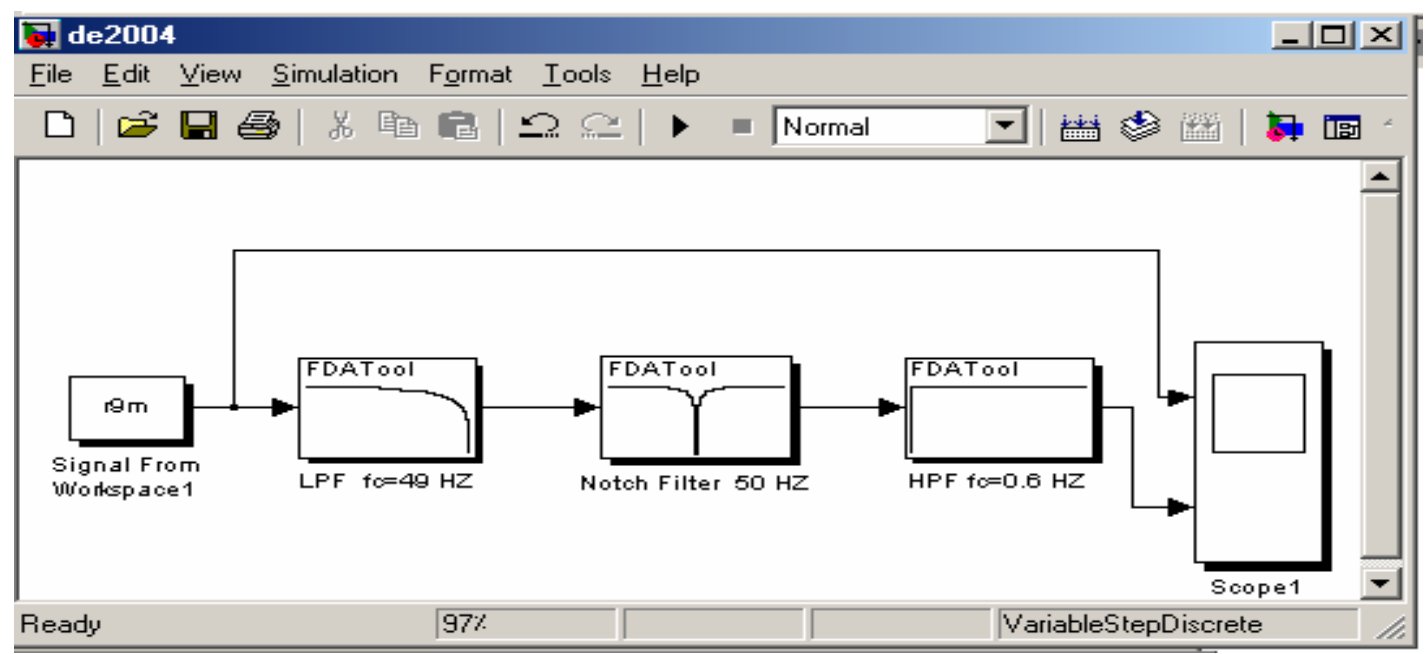

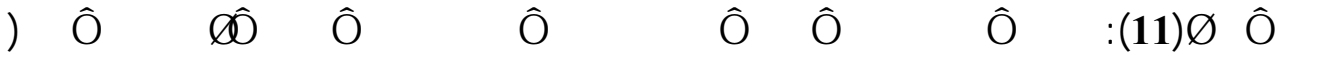

.Matlab ŝ̉ithimulink

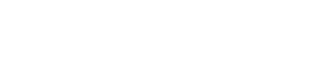

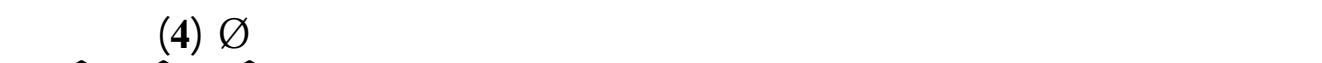

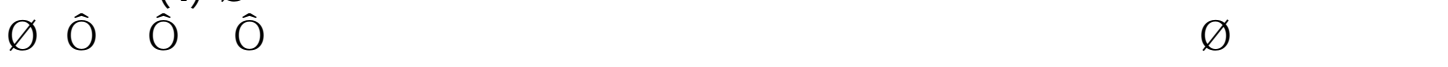

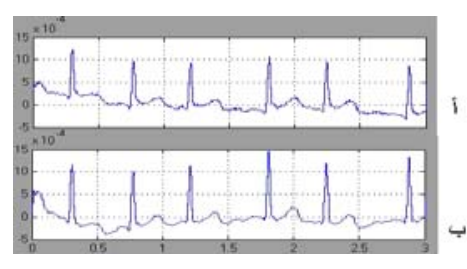

$-2-$

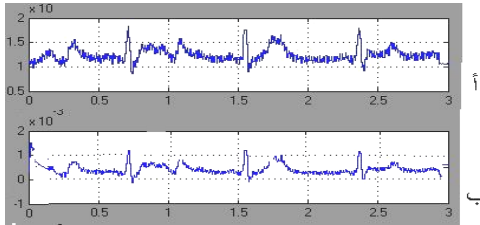

$-4-$

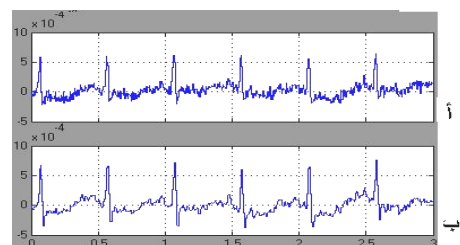

$-1-$

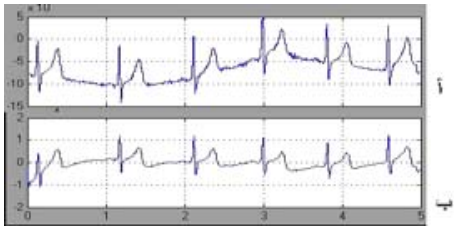

$-3-$

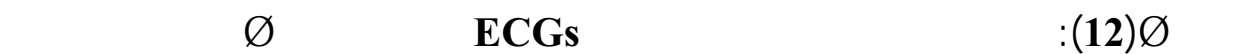

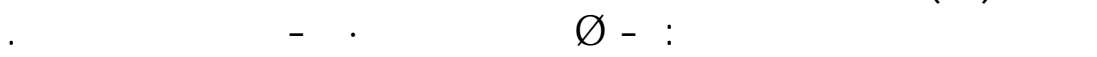

Cீ)

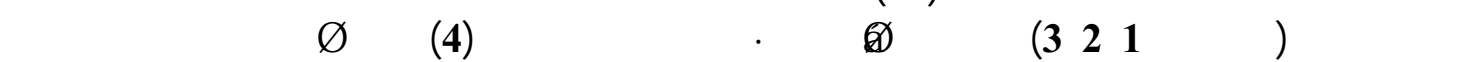

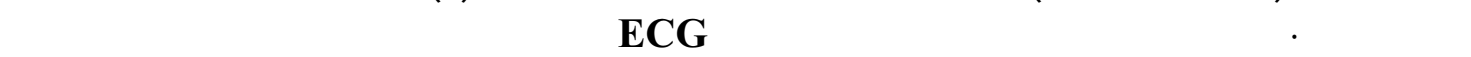

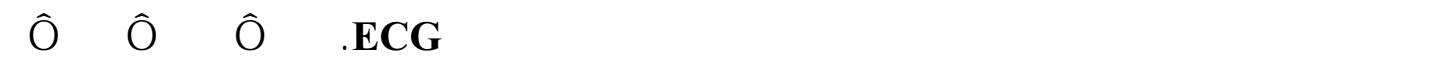

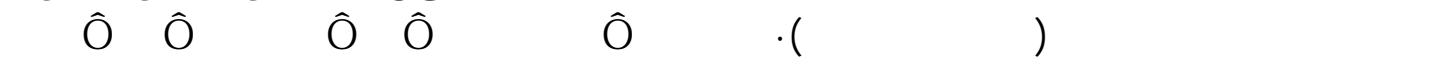




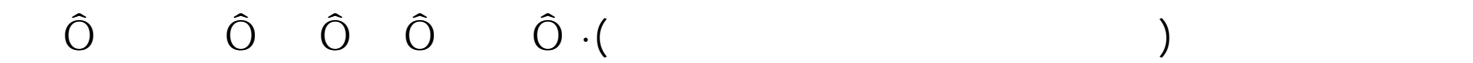

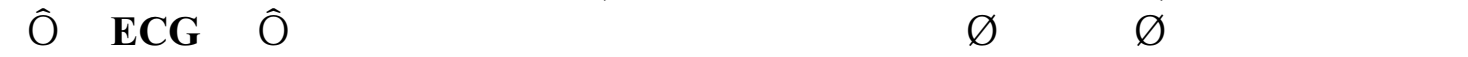

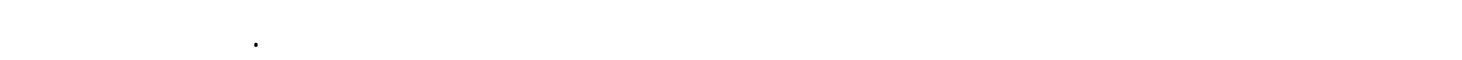

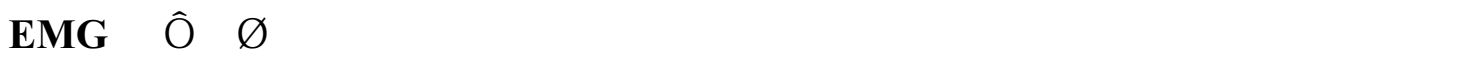

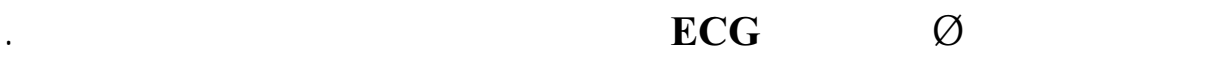

\section{REFERENCES}

tt'eooooo UJiz

[1]: Ben Ellis, "Pc-Based ECG Monitoring and Analysis Using web July24, 1998, the Note Application Instruments, National BioBenchTM", at

site

http://

www.zone.ni.com/devzone/conceptd.nsf/webmain/958FAC9BE8DAE 5862568690079BAEA/\$File/AN124

[2]: Martin J.Burke and Denis T.Gleeson,"A Micropower Dry Electrode ENGINEERING, ECG preamplifier" , IEEE TRANS. ON BIOMEDICAL VOL.47, NO.2, FEBRUARY 2000, the web site at http://www.cae.wisc.edu/ meditate/paper/burke.pdf

[3]:Christopher M.Tenedero ,Mary Ann D.Raya and Luis G.Sison, "Design and Implementation of a Single-channel ECG Amplifier With DSP Post Processing in Matlab ",University of the Philippines, Diliman, Quezon City, 2002, available via internet from the author at pet11tenedero@yahoo.com

[4]: Bosch and Eckart Hartmnn,"ECG Front-End Design is simplified with Microconverter", November 2003, the web site at http://www.analog.com/analogdialogue http://www.ece.arizona.edu/ barton/ECG.doc 
[5]:Analog Device, Precision Instrumentation Amplifier, AD524

www.analog.com/UploadedFiles/Data_Sheets771784793AD524_e.pdf [6]: Bruce Carter,"An Audio Collection, part 2", Analog Application Journal, February 2001: http:// www.lapsi.eletro.ufrgs.br/.../App $\% 20$ Notes/Texas \%20

20Analog\%20Applications \%20Journal/pdf-separados/feb_10.pdf

[7]: Sound Cards, AOpen Inc., 2003, the web site at

www.aiyamicro.com/htm-oem-computer- http://

gear/mutimedia

[8]: The MathWorks, Inc "Data Acquisition Toolbox For Use with MATLAB", 2002, the web site at www.desi.iteso.mx/elec/labs/infra_soft_info/matlab/daqug.pdf [9]: The MathWorks, Inc "Filter Design Toolbox For Use with Matlab", 2002. 